\title{
Duplicated appendix with an acute abdomen in 16 years old female with both appendices inflamed: a rare case report
}

\author{
Shivakumar S. ${ }^{1}$, N. Uday Kumar ${ }^{2 *}$ \\ ${ }^{1}$ Department of General Surgery, JSS Hospital, Mysuru, Karnataka, India \\ ${ }^{2}$ Department of General Surgery, Chettinad Hospital and Research Institute, Chennai, Tamil Nadu, India
}

Received: 21 August 2020

Revised: 28 September 2020

Accepted: 02 October 2020

\author{
*Correspondence: \\ Dr. N. Uday Kumar, \\ E-mail: Uday73kumar43@gmail.com
}

Copyright: () the author(s), publisher and licensee Medip Academy. This is an open-access article distributed under the terms of the Creative Commons Attribution Non-Commercial License, which permits unrestricted non-commercial use, distribution, and reproduction in any medium, provided the original work is properly cited.

\begin{abstract}
Duplicated appendix is a rare congenital anomaly with incidence of 0.004-0.009\% and its mostly an incidental finding on table, when one of them is acutely inflamed and very rarely both of them can be inflamed as in this case. Report a case of young girl who presented with complain of pain abdomen and vomiting in the last 2 days. Clinically patient was diagnosed to have acute appendicitis and on laparoscopy, patient had duplicated appendix one at the ileocaecal valve and the other $2 \mathrm{~cm}$ away near the caecum with pus and faecolith. Histopathology confirmed appendicitis in the both appendices. Reported about this interesting rare case because even though the incidence of duplicated appendix is too low (0.004-0.009\%), should always search for the missed appendix in patients who underwent appendicectomy earlier and complains of severe pain in right iliac fossa. Aim was to report such a rare interesting case and give a small gentle reminder to the surgeons as duplicated appendix even though rare but still a possibility.
\end{abstract}

Keywords: Duplicated appendix, Appendicitis, Appendicectomy, Right iliac fossa

\section{INTRODUCTION}

Incidence of duplicated appendix varies from 0.004 $0.009 \%$, which was first described in 1892 by Picoli. Duplicated is a commonly missed and neglected diagnosis with life threatening consequences. If duplicated appendix is seen in children then see for other congenital anomalies of intestine, Genito-urinary abnormalities. Almost all cases were diagnosed incidentally and most of the duplicated appendix cases published in the literature were detected incidentally.,2 During an autopsy or laparoscopy or laparotomy, radiological studies such as abdominopelvic computed tomography (CT) for the diagnosis of acute appendicitis. $^{3,4}$

\section{CASE REPORT}

16 years old female came to the outpatient department with complains of pain abdomen in the last 2 days associated with nausea and vomiting. History of fever in the last 2 days. On examination patient had tenderness in right iliac fossa, mild guarding and rigidity present and was diagnosed to have acute appendicitis. Per rectal examination was normal. Blood investigations done showed increase leucocyte count 12,240 (neutrophils $81 \%$ ) and others were within normal limits. Patient was planned for laparoscopic appendicectomy was done and intraoperatively omentum was seen adhering to the appendix, adhesions released and 2 tubular structures seen and surprisingly both were appendix. In which one appendix was seen inflamed with discharge of pus and fecolith and the other appendix was also inflamed. Mesoappendix of each appendix was divided and both the appendix were ligated at the base with 2-0 vicryl and cut. 
The second appendix was detected retrocaecal, $2 \mathrm{~cm}$ from the first one. Later thoroughly checked for any other intestinal anomalies and Meckel's diverticulum as the patient had duplication of appendix. Both the specimens were sent for histopathological examination. Histopathological examination of the surgical specimen confirmed the intraoperative findings of appendiceal duplication as well as findings of acute inflammation in both the appendix. The patient had an uneventful recovery and she was discharged on the second postoperative day. Patient is on regular follow up.

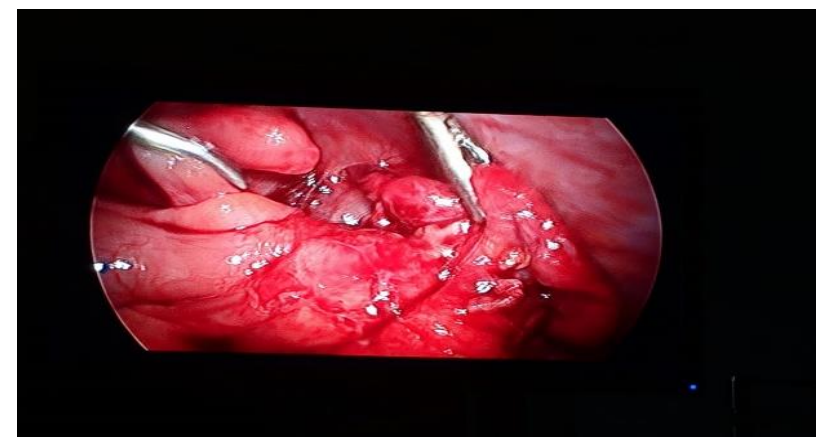

Figure 1: Laparoscopic view of duplicated appendix.

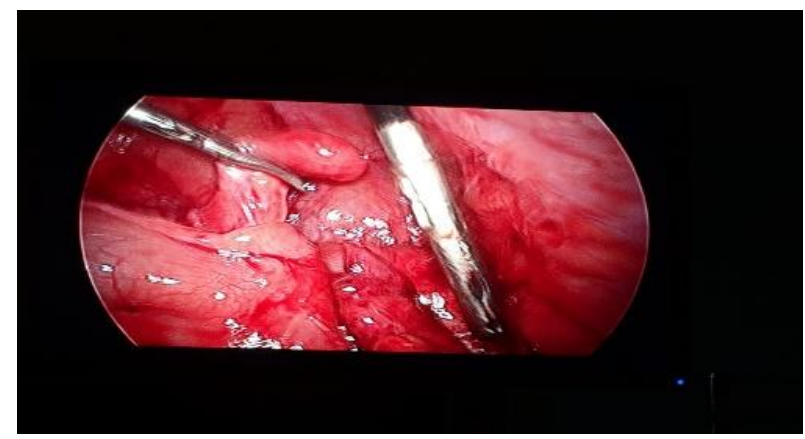

Figure 2: One appendix being held by atraumatic grasper and the other appendix on right side below the grasper.

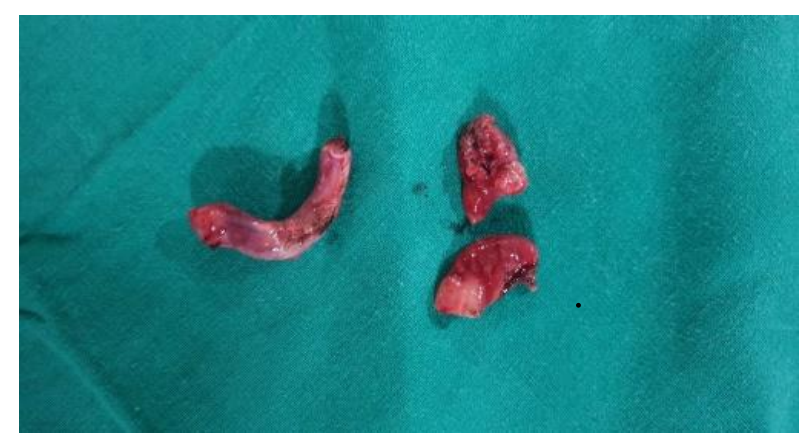

Figure 3: Duplicated appendix after surgery.

\section{DISCUSSION}

The appendix (or vermiform appendix) is a blind tube with average length of $7.5-10 \mathrm{~cm}$. Most common is retrocaecal in position, in $30 \%$ it is pelvic in position and retroperitoneal in $7 \%$ of population. Appendix is supplied by appendiceal artery, which is a branch of ileo-colic artery.

Duplicated appendix is a rare congenital anomaly which is usually discovered incidentally during surgery for appendicitis. Its incidence is $0.004-0.009 \%$. Even though duplicated appendix is uncommon they have clinical and medico-legal significance. It is also reported in a child who had appendectomies performed twice in a 5-month period.

The cave-wall bridge classification divides appendix duplications into three types ${ }^{5,6}$ : Type A-Single caecum with a normally localized appendix showing partial duplication. Type B-Single caecum with two complete appendices subdivided into two further, type B1 ('birdlike type')- Two appendices located symmetrically on both sides of the ileocaecal valve, mimicking the normal arrangement in birds, type B2 ('taenia coli' type)-One appendix originates from caecum at the typical site, and the second branches at varying distances along the taenia of first appendix, type C-Double caecum, each bearing its own appendix.

Some authors have incidentally detected at surgery complex presentation of appendix like horse shoe appendix and triple appendix..$^{7,8}$ When congenital anomalies of the appendix are detected in childhood see for other congenital anomalies as they are mostly associated with intestinal, genital, urinary or bone anomalies, seen most often in conjunction with type B1 and C duplications. ${ }^{9}$

\section{CONCLUSION}

Appendix duplication is a congenital anomaly that should not be excluded in abdominal pain cases. Duplication of appendix even though rare and diagnosing it preoperatively is difficult, surgeons should keep in mind about the possibility of duplication of appendix in a patient presenting with acute appendicitis and intraoperatively normal appendix which can lead to serious complications if the diagnosis is missed, so all the surgeons should be aware of duplication of appendix. Therefore, even in patients with a history of appendicectomy, the possibility of appendix duplication should not be overseen when the patient has pain in the right iliac fossa and clinical findings are favouring appendicitis.

Funding: No funding sources Conflict of interest: None declared

Ethical approval: Not required

\section{REFERENCES}

1. Şahin ST, Erhan Y, Aydede H. Double acute appendicitis in appendical duplication. Ulus Travma Acil Cerrahi Derg. 2013;19(1):83-5. 
2. Canbay E, Akman E. Appendix perforation in appendix duplication in a man: a case report. J Med Case Rep. 2011;5:162.

3. Erdoğan O, Arıcı C, Çolak T. Appendiks duplikasyonu: olgu sunumu. Ulus Travma Acil Cerrahi Derg. 2000;1:66-8.

4. Mann NS. Sandhu KS. Peppermint oil in irritable bowel syndrome: systematic evaluation of 1634 cases with meta-analysis. Int Med J. 2012;19:5-6.

5. Cave AJE. Appendix vermiformis duplex. J Anatomy. 1936;70(2):283-92.

6. Wallbridge PH. Double appendix. $\mathrm{Br} \mathrm{J}$ Surg. 1963;50:346-7.
7. Mesko TW, Lugo R, Breitholtz T. Horseshoe anomaly of the appendix: a previously undescribed entity. Surg. 1989;106(3):563-6.

8. Uriev L, Maslovsky I, Mnouskin Y, Ben-Dor D. Triple-barreled type of appendiceal triplication. Ann Diagno Pathol. 2006;10(3):160-1.

9. Sobhian B, Mostegel M, Kunc C, Karner J. Appendix vermiformis duplex (a rare surprise). Wien Klin Wochenschr. 2005;117(13-14):492-4.

Cite this article as: Shivakumar S, Kumar ND. Duplicated appendix with a acute abdomen in 16year-old female with both appendices inflamed-a rare case report. Int Surg J 2020;7:3782-4. 